\title{
Natural Resistance to HIV Infection: the Vif-APOBEC Interaction Michael H Malim*‡
}

\author{
Address: King's College London \\ Email: Michael H Malim* - michael.malim@kcl.ac.uk \\ * Corresponding author ‡Presenting author
}

from 2005 International Meeting of The Institute of Human Virology

Baltimore, USA, 29 August - 2 September 2005

Published: 8 December 2005

Retrovirology 2005, 2(SuppI I):SI44 doi:I0.II86/I742-4690-2-SI-SI44

Recent work has demonstrated that members of the APOBEC family of cellular polynucleotide cytidine deaminases - most notably APOBEC3G and APOBEC3F - are potent inhibitors of HIV infection. In the absence of the viral Vif protein, these two DNA editing enzymes are encapsidated by budding virus particles and then catalyse the deamination of cytidine $(C)$ to uridine $(U)$ in negative sense reverse transcripts. This results in guanosine $(G)$ to adenosine (A) hypermutation of viral plus stranded cDNA and reduced accumulations of viral DNA. Unexpectedly, recent structure-function analyses of APOBEC3G have revealed that substantial anti-viral phenotypes can be achieved in the absence of cytidine deamination. The relative contributions of these editing and editing-independent activities to the inhibition of infection in the absence of Vif remain to be defined. One view of the opposing functions of Vif and the APOBEC $3 \mathrm{G} / \mathrm{F}$ proteins during natural infection is that they are in are in "conflict" with each other. Accordingly, increases in APOBEC protein function or levels, or interference with Vif function, could culminate in enhanced anti-viral function and/or mutation rates. Whether such shifts in the Vif/APOBEC balance can influence the natural history of HIV infection is a critical future question, and supporting evidence would further indicate that perturbing this balance deserves consideration as a future therapeutic strategy. 